\title{
IONOSPHERIC SCINTILLATION MODEL LIMITATIONS AND IMPACT IN GNSS-R MISSIONS
}

\author{
A. Camps ${ }^{1,4}$, G. Gonzalez.Casado ${ }^{2}$, J. M. Juan $^{2}$, J. Sanz $^{2}$, H. Park ${ }^{3,4}$, J. Barbosa $^{5}$ \\ ${ }^{1}$ CommSensLab-UPC Unidad María de Maeztu - Dep. of Signal Theory and Communications, \\ ${ }^{2} \mathrm{gAGE}$ - Research Group of Astronomy and Geomatics, \\ ${ }^{3}$ Department of Physics, \\ Universitat Politècnica de Catalunya UPC Campus Nord, E-08034 Barcelona, Spain \\ ${ }^{4}$ IEEC/CTE-UPC, UPC Campus Nord, E-08034 Barcelona, Spain \\ ${ }^{5} \mathrm{RDA}$ - Research and Development in Aerospace GmbH, Zürich, Switzerland \\ E-mail: camps@tsc.upc.edu
}

\begin{abstract}
The ionosphere impacts radio-wave propagation, notably up to a few GHz. The main applications impacted by the ionosphere are GNSS positioning and timing, Earth Observations (especially low frequency SAR missions e.g. BIOMASS, and GNSS-R), and Space Weather. While most effects can be compensated by using dual-frequency receivers and circular polarization antennas, ionospheric scintillation (rapid intensity and phase fluctuations) cannot. Climatological models for the mean stable part of the electron density in the ionospheric layers (e.g. IRI or NeQuick) or for the magnetic field (e.g. WMM) have significantly improved in the past years. However, models of the inhomogeneous part, responsible for scintillation, can be improved, since they are based on relatively old data (e.g. WBMOD), or climatological inputs are limited to properly characterize all latitudes and solar conditions (e.g. GISM or WAM). This study first assesses the goodness of GISM, the model adopted by the ITU-R, by comparing GISM predictions and measured scintillation data. Then, the impact of measured intensity and phase scintillation on TDS-1 GNSS-R data is illustrated.
\end{abstract}

Index Terms - GNSS-R, ionosphere, scintillation, models.

\section{INTRODUCTION}

The ionosphere impacts radio-wave propagation, notably up to a few GHz. While propagating through the ionosphere radio waves may suffer a rotation of the polarization plane ("Faraday rotation") due to its interaction with the ionized medium in the Earth's magnetic field along the path, a group delay and phase advance of the signal due to the total electron content (TEC) accumulated along the path, a rapid variation of the signal's amplitude and phase ("scintillations") due to small-scale irregularities, a change in the apparent direction of arrival ("refraction"), and Doppler effects due to nonlinear polarization rotations and time delays [1]. The main applications impacted by ionospheric effects are GNSS positioning and timing, Earth Observations (low frequency SARs and GNSS-R), and Space Weather.

Ionospheric scintillations occur mostly in equatorial and high latitude regions, and their behavior is different. Equatorial scintillation occur around $\pm 20^{\circ}$ of latitude of the magnetic equator, after sunset, and before midnight, and are produced by convective plasma processes. High latitude scintillations occur in polar region, mostly during the dark months at all local times, while auroral zones are observed during the nighttime. Lastly, mid latitude scintillations occur as an extension of phenomena occurring at equatorial and auroral latitudes, or due to an intense sporadic E layer during the daytime. While in Equatorial regions amplitude and phase scintillations occur, in high latitude regions phase scintillation is dominant. Amplitude ionospheric scintillation is usually characterized by the $\mathrm{S}_{4}$ parameter, while phase scintillation by its standard deviation or $\sigma_{\varphi}$, and the slope of the power spectrum of signal phase $(p)$.

Ionospheric climatological models for the stable part of the electron density in the ionospheric layers or for the Earth's magnetic field have been significantly improved in the past years, but models for the inhomogeneous part, responsible for scintillation effects, are still far from precise. GISM (Global Ionospheric Scintillation Model) [2] is the model adopted by ITU-R to predict Ionospheric Scintillation [3]. It is known that it fails to predict high latitude scintillation. SCIONAV is an updated model trying to overcome some of the limitations of GISM (weak phase scintillation estimates in high latitudes, presence of equatorial bubbles and depletions) was presented in [4], as an outcome of the project "Improved Modelling of Short and Long Term Characteristics of Ionospheric Disturbances During Active Years of the Solar Cycle," ESTEC contract 4000115300/15/NL/AF.

In this work, the goodness of GISM and SCIONAV models is evaluated at different monitoring stations in terms of the errors in the prediction of the $\mathrm{S}_{4}, \sigma_{\varphi}$, and $p$, as a function of the ionospheric activity (high, medium, low or quiet), their location, and the azimuth and elevation angles. To do that, a suitable data set of GNSS scintillation was gathered and compared statistically with respect to the model outputs. Then, GNSS-R observables (fluctuations of the peak of the Delay-Doppler Map) from TDS-1 data collocated as much as possible in time and space [5] were analyzed to assess the impact in GNSS-R missions [6]. 


\section{DATA COLLECTION}

The collected data are the following scintillation parameters and ionospheric activity indicators: $\mathrm{S}_{4}, \sigma_{\varphi}, p$-slope (slope of power spectra of phase scintillation from $\mathrm{L}_{1}$ ), ROTI ${ }^{1}$ (Rate of TEC Index, being TEC the Total Electron Content) from the geometry-free combination, and AATR ${ }^{2}$.

The data were obtained from different types of ground receivers belonging to different networks:

- IGS stations, high-rate receivers $(1 \mathrm{~Hz})$,

- MONITOR-ESA stations, ISMR receivers $(50 \mathrm{~Hz})$, and

- Stations from the Space Weather Service of Australian government (SWSAu), ISMR receivers $(50 \mathrm{~Hz})$.

Part of the data collected from ISMR receivers has been used to verify the reliability of data from $1-\mathrm{Hz}$ stations, that is, as a subset for quality control of future reference data sets. However, another part has been also used to support model validation, particularly for $p$-slope and for $\mathrm{S}_{4}$ in the equatorial region of South-America.

Geodetic data at $1-\mathrm{Hz}$ may seem, in principle, to be affected by larger restrictions and inaccuracies, but they have some important advantages, namely:

- They are far more abundant than ISMR data, both in the space and time domains.

- They have been carefully processed with an accurate methodology that allows selection of the more reliable data and, in particular, removes cycle slips (even single ones) with high reliability from the measurements, rendering data sets with a good quality control.

ISMR data at $50-\mathrm{Hz}$ may appear as more accurate to trace scintillation phenomena, but they have a main disadvantage: - Coming from external sources (MONITOR and SWSAu), with almost no information on the pre-processing and/or quality controls applied, which may be different.

In short, ISMR data are not always the best choice, and that it may also have some limitations that can be compensated with the proper processing.

Also, only GPS data were used for model validation, with a minimum elevation angle of $40^{\circ}$ for model validation. The list of consolidated GNSS-R scenarios is shown in Table 1.

\section{IONOSPHERIC SCINTILLATION MODEL VALIDATION}

The general behavior of the GISM and SCIONAV models is compared here with the data. Plots are divided into geographical regions, but they are not into scintillation categories. Low Latitude data from America and Asia/Africa regions are merged as no different behavior was found from this analysis.

Figure 1 shows the $\mathrm{S}_{4}$ Probability Density Functions (PDF) from GISM/SCIONAV (neglecting the bubbles and depletions, SCIONAV has the same output for $\mathrm{S}_{4}$ as GISM). Note that $\mathrm{S}_{4}$ is not properly characterized, as it is overpredicted at equatorial regions and under-predicted at polar

\footnotetext{
${ }^{1}$ ROTI is the standard deviation of the ROT (Rate of TEC) on 5 min intervals, and ROT is the TEC derivative in 1 min intervals.
}

latitudes. At mid latitudes the behaviour is noisy, a bit erratic. The dependence with ROTI and LT (Figs. 2, 3) is well captured, notably at equatorial regions, but it is not so good at mid-latitudes and polar regions.

Table 1 - List of consolidated GNSS-R scenarios

\begin{tabular}{|c|c|c|c|c|c|c|c|}
\hline \multirow[b]{2}{*}{ ID label } & \multicolumn{4}{|c|}{ Time Period } & \multicolumn{3}{|c|}{ Scenario description } \\
\hline & YYYY & MM & DoM & UT (h) & $\begin{array}{c}\text { Iono } \\
\text { activity }\end{array}$ & $\begin{array}{l}\text { Solar } \\
\text { activity }\end{array}$ & $\begin{array}{l}\text { Com- } \\
\text { ments }\end{array}$ \\
\hline \multirow{2}{*}{\multicolumn{8}{|c|}{$\begin{array}{l}\text { Type } 1 \text { Scenarios } \\
\text { Polar Can and High Latitude Regions }\end{array}$}} \\
\hline & & & ude Regi & ons & & & \\
\hline 1-HLT-1 & 2015 & 4 & 16 & {$[19,24]$} & Moderate & High & \multirow{2}{*}{$\begin{array}{l}\text { Moderate } \\
\text { geomag. } \\
\text { storm }\end{array}$} \\
\hline 1-HLT-2 & 2015 & 9 & 9 & {$[3,14]$} & Moderate & High & \\
\hline \multicolumn{8}{|c|}{$\begin{array}{ll}\text { Low Equatorial Regions } \\
\end{array}$} \\
\hline 1-LEQ-1 & 2015 & 2 & 27 & \multirow[b]{2}{*}{$\begin{array}{l}{[19,24]} \\
\&[0,2]\end{array}$} & \multirow[b]{2}{*}{ High } & \multirow[b]{2}{*}{ High } & \multirow{2}{*}{$\begin{array}{l}\text { Highest } \\
\text { scintill. } \\
\text { with TDS } \\
\text { data }\end{array}$} \\
\hline 1-LEQ-2 & 2015 & 3 & 16 & & & & \\
\hline \multicolumn{8}{|c|}{ Type 2 Scenarios } \\
\hline \multicolumn{8}{|c|}{ Polar Cap and High Latitude Regions } \\
\hline 2-HLT-1 & 2015 & 3 & 24,25 & {$[9,19]$} & Mid-high & High & \\
\hline \multicolumn{8}{|c|}{ Low Equatorial Regions } \\
\hline 2-LEQ-1 & 2015 & 5 & $\begin{array}{c}10,11 \\
12\end{array}$ & {$[10,20]$} & High & High & $\begin{array}{l}\text { Mod. } \\
\text { scintill. }\end{array}$ \\
\hline \multirow{2}{*}{\multicolumn{8}{|c|}{$\begin{array}{l}\text { Type } 3 \text { Scenarios } \\
\text { Polar Cap and High Latitude Regions } \\
\end{array}$}} \\
\hline & & & & & & & \\
\hline 3-HLT-1 & 2015 & 4 & 8 & {$[13,24]$} & 3 & High & $\begin{array}{l}\text { Mostly low } \\
\text { activ. }\end{array}$ \\
\hline 3-HLT-2 & 2015 & 4 & 25 & {$[16,23]$} & 3 & High & $\begin{array}{l}\text { Mostly low } \\
\text { activ. }\end{array}$ \\
\hline 3-HLT-3 & 2016 & 7 & 23,24 & $\begin{array}{l}12 \mathrm{~h} \text { of } 23 \\
15 \mathrm{~h} \text { of } 24\end{array}$ & 3 & Low & $\begin{array}{c}\text { Nearly quiet } \\
\text { iono. }\end{array}$ \\
\hline \multicolumn{8}{|c|}{ Low Equatorial Regions } \\
\hline 3-LEQ-1 & 2015 & 6 & 27,29 & $\begin{array}{c}{[0,6]} \\
\& \\
{[21,24]}\end{array}$ & 3 & Medium & $\begin{array}{l}\text { Low activ.\& } \\
\text { scintill. }\end{array}$ \\
\hline 3-LEQ-2 & 2015 & 7 & $\begin{array}{c}21,22, \\
23\end{array}$ & {$[0,6]$} & 3 & Medium & $\begin{array}{l}\text { Quiet iono., } \\
\text { no scintill. }\end{array}$ \\
\hline
\end{tabular}
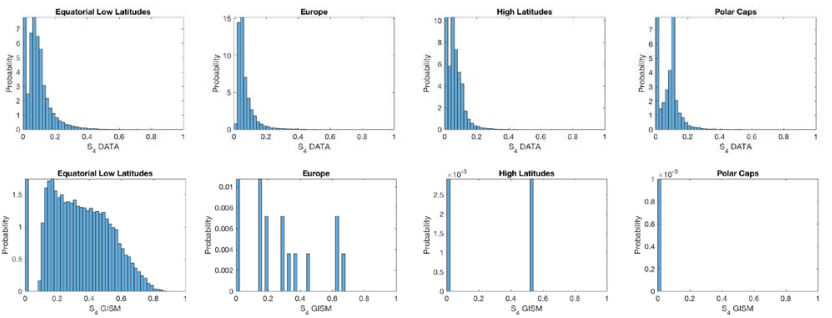

Fig. 1 Regional $\mathrm{S}_{4} \mathrm{PDF}$, from the data (top), and from GISM and SCIONAV models (bottom). Note: SCIONAV has the same output as GISM.
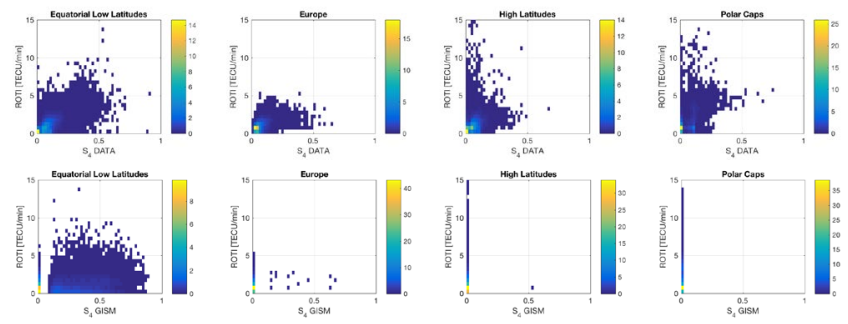

Fig. 2 Regional $\mathrm{S}_{4}$ vs ROTI from the data (top), and from GISM and SCIONAV models (bottom). Note: SCIONAV has the same output as GISM.

${ }^{2}$ AATR is the derivative of the STEC (slant TEC) divided by the square of the obliquity factor. 


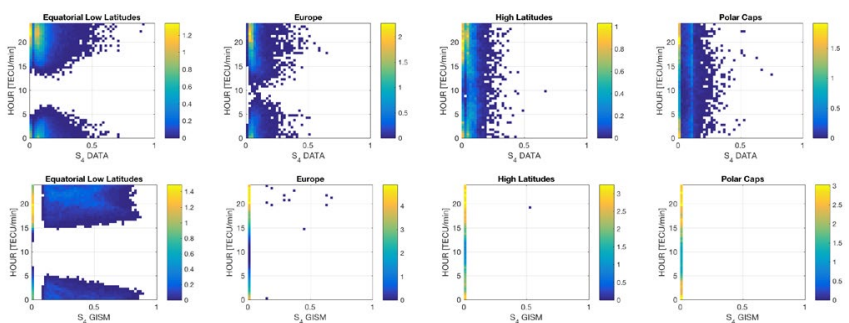

Fig. 3 Regional $\mathrm{S}_{4}$ vs LT from the data (top), and from GISM and SCIONAV models (bottom). Note: SCIONAV has the same output as GISM.
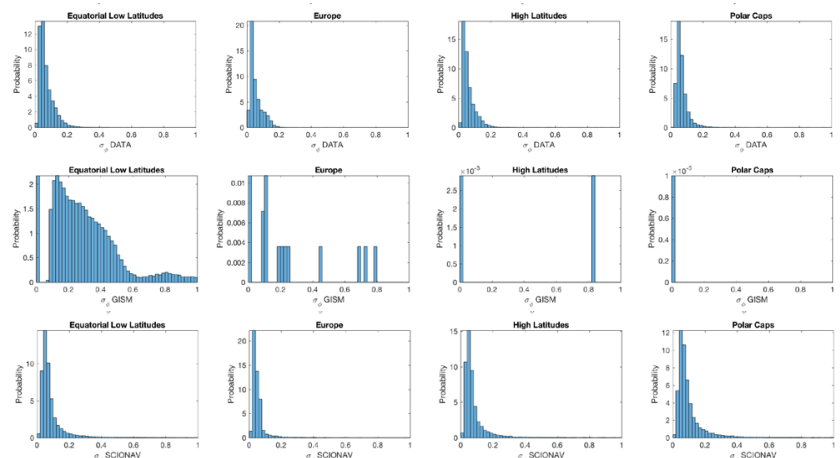

Fig. 4 Regional $\sigma_{\phi}$ PDF from the data (top), from GISM (center), and SCIONAV (bottom) models.
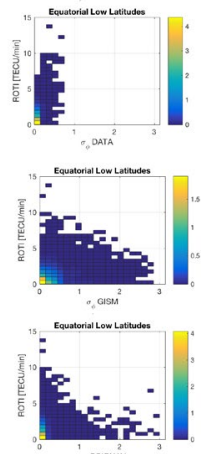

$\operatorname{sichavin}^{2}$
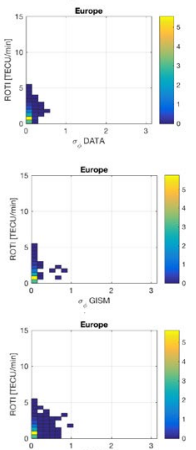

$\operatorname{scosen}^{2}$
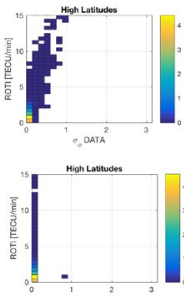

${ }^{\prime} \operatorname{sish}^{2}$

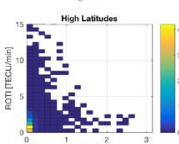

sconing
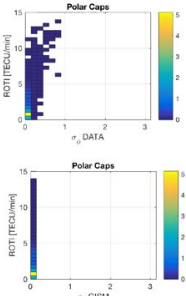

$\operatorname{cosin}^{2}$

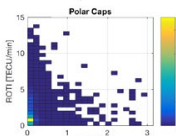

Fig. 5 Regional $\sigma_{\phi}$ vs ROTI from the data (top), from GISM (center), and SCIONAV (bottom) models.

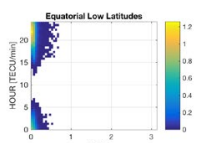

. DARÁ

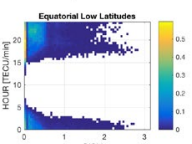

$\operatorname{GSM}^{2}$
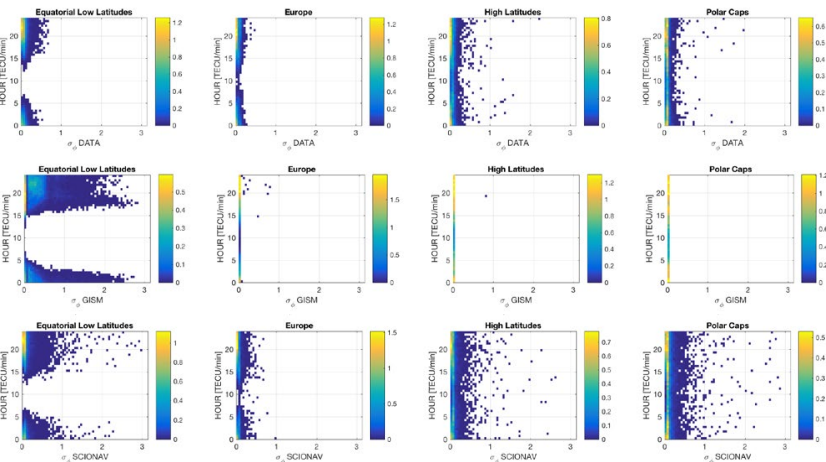

Fig. 6 Regional $\sigma_{\phi}$ vs LT from the data (top), from GISM (center), and SCIONAV (bottom) models.
Figure 4 shows the $\sigma_{\phi}$ PDF from GISM, from SCIONAV, and from the measured data. While GISM again overestimates $\sigma_{\phi}$ at equatorial regions, under-predicts at polar latitudes, and has a noisy behaviour at mid latitudes, SCIONAV model shows an outstanding agreement. The dependence with ROTI and LT (Figs. 5, 6) is also well captured at all regions by SCIONAV, although only at equatorial regions by GISM.

Table 2 and 3 show the error analysis for $S_{4}$ and $\sigma_{\phi}$ in terms of mean and root mean square (RMS) errors. For $\sigma_{\phi}$ phase scintillation shows a significant improvement of SCIONAV over GISM for all regions in terms of the mean, and the RMS for equatorial regions.

Table $2-\mathrm{S}_{4}$ error analysis

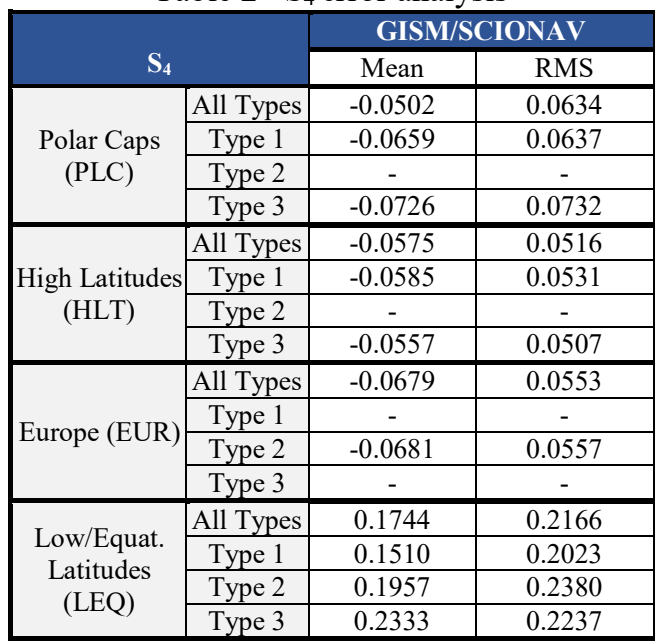

Table $3-\sigma_{\phi}$ error analysis

\begin{tabular}{|c|c|c|c|c|c|}
\hline \multirow{2}{*}{$\sigma_{\phi}$} & & \multicolumn{2}{|c|}{ GISM } & \multicolumn{2}{|c|}{ SCIONAV } \\
\hline & & Mean & RMS & Mean & RMS \\
\hline \multirow{4}{*}{$\begin{array}{l}\text { Polar Caps } \\
\text { (PLC) }\end{array}$} & All Types & -0.0686 & 0.0542 & 0.0402 & 0.1798 \\
\hline & Type 1 & -0.0791 & 0.0742 & 0.0599 & 0.2274 \\
\hline & Type 2 & - & - & - & - \\
\hline & Type 3 & -0.0650 & 0.0287 & 0.0284 & 0.1151 \\
\hline \multirow{4}{*}{$\begin{array}{l}\text { High Latitudes } \\
\text { (HLT) }\end{array}$} & All Types & -0.0625 & 0.0643 & 0.0206 & 0.1392 \\
\hline & Type 1 & -0.0937 & 0.1164 & 0.0563 & 0.2857 \\
\hline & Type 2 & - & - & - & - \\
\hline & Type 3 & -0.0502 & 0.0316 & 0.0082 & 0.0663 \\
\hline \multirow{4}{*}{ Europe (EUR) } & \begin{tabular}{|l|} 
All Types \\
\end{tabular} & -0.0541 & 0.0394 & -0.0055 & 0.0629 \\
\hline & Type 1 & - & - & - & - \\
\hline & Type 2 & -0.0522 & 0.0375 & -0.0033 & 0.0610 \\
\hline & Type 3 & - & - & - & - \\
\hline \multirow{4}{*}{$\begin{array}{l}\text { Low/Equat. } \\
\text { Latitudes } \\
\text { (LEQ) }\end{array}$} & All Types & 0.2133 & 0.3410 & 0.0182 & 0.1356 \\
\hline & Type 1 & 0.1623 & 0.2437 & 0.0484 & 0.2190 \\
\hline & Type 2 & 0.4540 & 0.5875 & 0.0133 & 0.1085 \\
\hline & Type 3 & 0.2907 & 0.4184 & 0.0076 & 0.0808 \\
\hline
\end{tabular}

\section{IMPACT ON GNSS-R DATA}

In [6] the impact of ionospheric scintillation was analyzed. It was found that due to the way the data are processed (short coherent integration time 1-4 ms, followed by long incoherent averaging $\sim 1 \mathrm{~s}$ ), only ionospheric range errors and intensity scintillations are important. Since scintillation is a phenomenon that takes place only when coherent electromagnetic waves transverse the ionosphere, 
fluctuations of the peak of the Delay-Doppler Map (DDM) or Waveform may only be noticeable when the scattering takes place over a relatively flat surface, such as calm sea, ice, and some land surface.

Figure 7 shows the radar plots (azimuth = local azimuth, radius $=$ cosine of elevation angle) of the $S_{4}, \sigma_{\phi}$, and the standard deviation of the peak of the DDM ( $\sigma_{\text {DDMpeak }}$ ) computed in a moving window of 10 consecutive samples of 1 s. The largest DDM peak fluctuations ( $\left.\sigma_{\text {DDMpeak }}\right)$ occur around $\pm 20^{\circ}$ latitude, and the visual correspondence of the

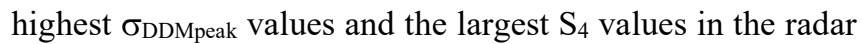
plots is also evident. Some large fluctuations are observed however, in regions illuminated nearly zenith by SBAS systems.
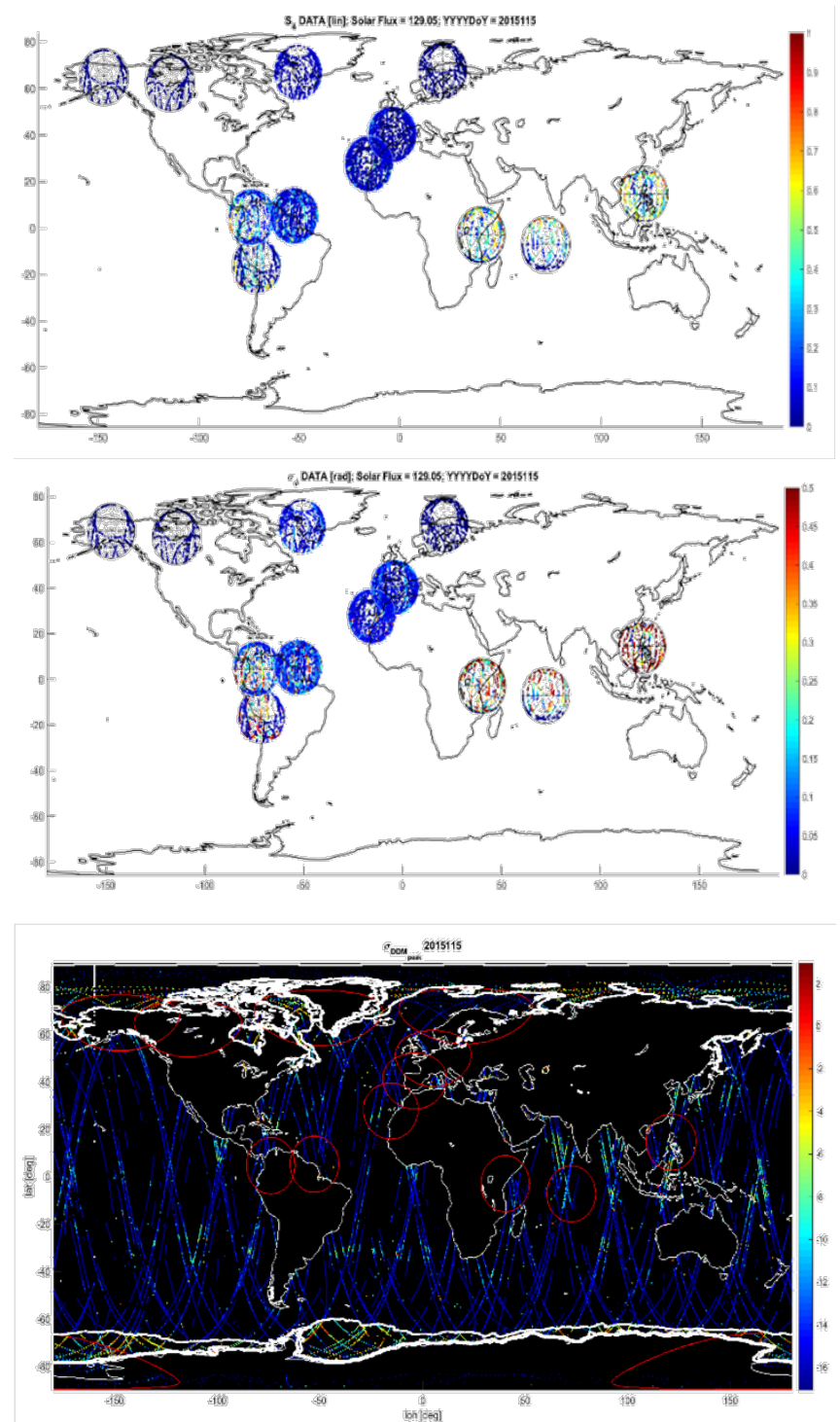

Fig. 7 Radar plots of $\mathrm{S}_{4}, \sigma_{\phi}$ and $\sigma_{\text {DDMpeak }}$ for the ground stations analyzed. Day: 2015115. High Ionospheric activity at mal2 and pimo stations.

\section{CONCLUSIONS AND FUTURE WORK}

It has been found that $\mathrm{S}_{4}$ is not properly modelled at high latitudes for GISM/SCIONAV, and it is overestimated at equatorial regions. Improved models are required. $\sigma_{\phi}$ is better modelled with SCIONAV for all regions, while it is overestimated by GISM at equatorial regions, and underestimated at polar regions. There is also an anomalous behavior of the modelled $p$ (slope of the phase scintillation spectrum), not shown in this extended abstract that has to be analyzed in further detail. Finally, bubbles and depletions may be included again in the SCIONAV model for a more detailed analysis.

Finally, the analysis of the fluctuations of the TDS-1 GNSSR DDM peak show strips of larger values around $\pm 20^{\circ}$ latitude, and in regions with high measured $\mathrm{S}_{4}$ values.

\section{ACKNOWLEDGMENTS}

This work was supported by ESA/ESTEC project 4000120868/17/NL/AF "Radio Climatology Models of the Ionosphere: Status and Way Forward," by the Spanish Ministry of Science, Innovation and Universities, "Sensing with Pioneering Opportunistic Techniques" SPOT, grant RTI2018-099008-B-C21, and by the Unidad de Excelencia Maria de Maeztu MDM-2016-0600. The authors want to thank the discussions held with Dr. J. Lemorton, V. Fabbro, A. Mainvis (ONERA), and Dr. R. Orús (ESA) in the frame of the project.

\section{REFERENCES}

[1] Recommendation ITU-R P.531-11 (01/2012) Ionospheric propagation data and prediction methods required for the design of satellite services and systems. Available online: https://www.itu.int/dms_pubrec/itu-r/rec/p/R-REC-P.531-11201202-S!!PDF-E.pdf (last visited January 24, 2019)

[2] Y Beniguel, "Global Ionospheric Propagation Model (GIM): a propagation model for scintillations of transmitted signals", Radio Sci., vol. 37, no. 3, May 2002.

[3] REPORT ITU-R P.2097 Transionospheric radio propagation The Global Ionospheric Scintillation Model (GISM), 2007. Available online: https://www.itu.int/dms_pub/itu-r/opb/rep/R-REP-P.2097-

2007-PDF-E.pdf (last visited January 24, 2019)

[4] A. Camps, J. Barbosa, M. Juan, E. Blanch, D. Altadill, G. González, G. Vazquez, J. Riba, R. Orús, "Improved modelling of ionospheric disturbances for remote sensing and navigation," 2017 IEEE International Geoscience and Remote Sensing Symposium (IGARSS), Fort Worth, TX, 2017, pp. 2682-2685.

[5] A. Camps, H. Park, J.M. Juan, J. Sanz, G. González-Casado, J. Barbosa, V. Fabbro, J. Lemorton, R. Orús, "Ionospheric Scintillation Monitoring Using GNSS-R?", Geoscience and Remote Sensing Symposium IGARSS 2018 - 2018 IEEE International, pp. 3339-3342, 2018.

[6] A. Camps, H. Park, G. Foti, C. Gommenginger, "Ionospheric Effects in GNSS-Reflectometry From Space", IEEE Journal of Selected Topics in Applied Earth Observations and Remote Sensing, vol. 9, no. 12, pp. 5851-5861, Dec. 2016. 